\title{
REVISITING THE ROLE OF AGGREGATION IN THE SETTLING OF COHESIVE FLOCS IN THE MARINE ENVIRONMENT
}

\author{
Ashish J. Mehta, Nutech Consultants, Gainesville, FL, mehtanutechinc@gmail.com \\ William H. McAnally, Dynamic Solutions, Knoxville, TN, whmcanally@dsllc.com \\ Farzin Samsami, Formerly at Heriot-Watt Univ., Edinburgh, UK, f.samsami@hw.ac.uk \\ Andrew J. Manning, Plymouth Univ.; HR Wallingford, UK, andymanning@yahoo.com
}

\section{INTRODUCTION}

The settling velocity is the single-most important property governing the transport of cohesive flocs in the marine environment. In that regard, the instantaneously changing diameter, density and shear strength of flocs are the defining properties which distinguish floc transport from that of cohesionless particles. Thus, consideration of aggregation, which includes the dynamics of floc growth and breakup due to floc-floc collisions as well as flowinduced shearing of flocs, is a critical component of floc transport modeling.

The representation of floc settling velocity $w_{s}$ as a function solids volume fraction $\phi$ and the turbulent flow shear rate $G$ results in a settling velocity distribution dependent on the ranges of $G$ and $\phi$ in the (local) natural environment. Figure 1 shows such a relationship for data from the Tamar estuary in the UK (Manning 2001). The curves are based on the empirical formula

$$
w_{s}=w_{s h}(G)\left(\frac{\phi}{\phi_{h}}\right)^{n_{0}} ; w_{s h}(G)=a_{0}\left(\frac{1+\lambda_{a} G}{1+\lambda_{b} G^{2}}\right) ; \frac{\phi}{\phi_{h}} \leq 1 ; G \geq 1 s^{-1}
$$

where $w_{s h}(G)$ is the peak settling velocity at the onset of hindered settling which occurs when $\phi>\phi_{h}, n_{0}=1 / 3$ is a representative value of the exponent of $\phi / \phi_{h}$, and $a_{0}, \lambda_{a}$ and $\lambda_{b}$ are sediment dependent constants independent of $G$. Note that Eq. 1 is not applicable to hindered settling. In Fig. 1 , for each $\phi$ the rising arm of the $w_{s}$-G curve indicates the dominance of floc growth over breakup at low values of $G$, and the falling arm implies the dominance of breakup at higher $G$ values. Additional data of this type are available from several European estuaries and, also, San Francisco Bay (Manning and Schoellhamer 2013).

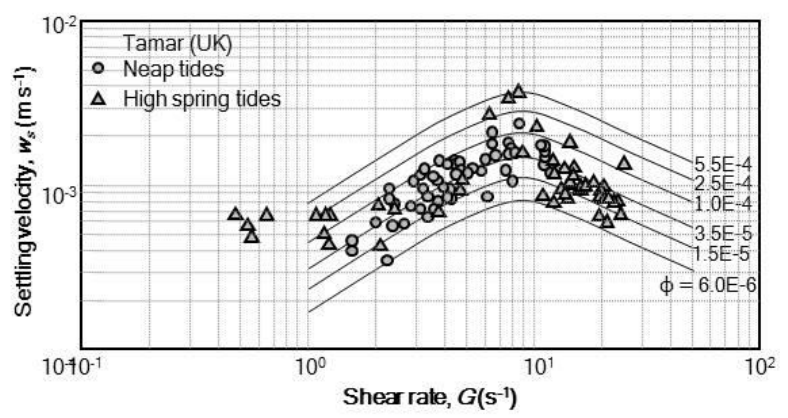

Figure 1 - Variation of $w_{s}$ with $G$ and $\phi$; data from Manning (2001); curves based on Eq. 1.

The present study is concerned with an effort to explain the physical underpinning of the five curves in Fig. 1 in terms of the application of the particle (floc) balance equation for the number concentration $N$ of flocs of suspended cohesive sediment in the turbulent flow environment.

\section{APPROACH}

The particle balance for $N$ is recast for flocs possessing fractal self-similarity represented by the fractal dimension $D$. Floc size distribution is represented by a discrete number of classes based on mass. Growth and breakup move mass up and down through the classes. The frequency of binary and ternary particle collisions due to Brownian motion, flow-induced shear and settling of flocs at different settling velocities is expressed in terms of simple statistical relationships.

This modeling approach has been previously shown to yield a physical explanation of time-dependent floc size distributions measured under laboratory conditions (McAnally 1999). The present study has made it possible to extend the analysis to prototype data such as those in Fig. 1 and similar plots for other selected estuaries. This makes it feasible to interpret Eq. 1 on a physical (non-empirical) basis.

A secondary objective is to examine purely analytic expressions for the settling velocity (of flocs of given fractal dimension $D$ ) as a function of $G$ and $\phi$. Based on simplifications of the particle balance two such expressions are considered, one in which floc growth is balanced by breakup due to flow shear, and the other in which breakup results from ternary collisions treated as two consecutive binary collisions.

\section{OBSERVATIONS}

It is shown that the behavior of Eq. 1 applied to $w_{s}-G$ data from selected estuaries can be reproduced based on the parameterization of floc density as a function of diameter and floc shear strength. The analytic expressions are shown to have limited applicability due to the simplified particle balance upon which they are based. The limits of applicability are identified by resorting to modeling the original (i.e. non-simplified) particle balance.

\section{REFERENCES}

Manning, A.J. (2001). A study of the effects of turbulence on the properties of flocculated mud, PhD Thesis, Univ. of Plymouth, Plymouth, UK.

McAnally, W.H. (1999). Transport of fine sediments in estuarial waters, PhD Thesis, Univ. of Florida, Gainesville.

Manning, A.J., Schoellhamer, D.H. (2013). Factors controlling floc settling velocity along a longitudinal estuarine transect, Mar. Geol., doi:10.1016/j.margeo.2013. 06.018. 